No. $2005-03$

THE BIRD CORE FOR MINIMUM COST SPANNING TREE PROBLEMS REVISITED: MONOTONICITY AND ADDITIVITY ASPECTS

By Stef Tijs, Stefano Moretti, Rodica Branzei, Henk Norde

December 2004

ISSN 0924-7815 


\title{
The Bird core for minimum cost spanning tree problems revisited: monotonicity and additivity aspects
}

\author{
Stef Tijs ${ }^{1}$, Stefano Moretti ${ }^{2}$, Rodica Branzei $^{3}$, Henk Norde ${ }^{4}$
}

December 21, 2004

\begin{abstract}
A new way is presented to define for minimum cost spanning tree (mcst-) games the irreducible core, which is introduced by Bird in 1976. The Bird core correspondence turns out to have interesting monotonicity and additivity properties and each stable cost monotonic allocation rule for mcst-problems is a selection of the Bird core correspondence. Using the additivity property an axiomatic characterization of the Bird core correspondence is obtained.
\end{abstract}

Key-words: cost allocation, minimum cost spanning tree games, Bird core, cost monotonicity, cone additivity.

JEL classification: C71.

\section{Introduction}

One of the classical problems in Operations Research is the problem of finding a minimum cost spanning tree (mcst) in a connected network. For algorithms solving this problem see Kruskal (1956) and Prim (1957). Claus and Kleitman (1973) discuss the problem of allocating costs among users in

\footnotetext{
${ }^{1}$ Department of Mathematics, University of Genoa, Italy and CentER and Department of Econometrics and Operations Research, Tilburg University, The Netherlands.

${ }^{2}$ Department of Mathematics, University of Genoa and Department of Environmental Epidemiology, National Cancer Research Institute of Genoa, Italy.

${ }^{3}$ Faculty of Computer Science, "Alexandru Ioan Cuza" University, Iasi, Romania.

${ }^{4}$ CentER and Department of Econometrics and Operations Research, Tilburg University, The Netherlands.
} 
a minimum cost spanning tree. This inspired independently Bird (1976) and Granot and Claus (1976) to construct and use a cooperative game to tackle this cost allocation problem.

In the seminal paper of Bird (1976) a method is indicated how to find a core element of the minimum cost spanning tree game (mcst game) when a minimum cost spanning tree is given. Further he has introduced, using a fixed mcst, the irreducible core of an mcst game, which is a subset of the core of the game, and which we will call in this paper the Bird core. The Bird core is central in this paper. First, we will give a new "tree free" way to introduce the Bird core by constructing for each mcst-problem a related problem, where the weight function is a non-Archimedean semimetric. The Bird core correspondence turns out to be a crucial correspondence if one is interested in stable cost monotonic allocation rules for mcst-problems. In fact, the Bird core is the "largest" among the correspondences which are cost monotonic and stable. The Bird core has also an interesting additivity property i.e. the Bird core correspondence is additive on each Kruskal cone in the space of mcst-problems with a fixed number of users. The additivity on Kruskal cones can be used to find an axiomatic characterization of the Bird core correspondence.

The outline of the paper is as follows. Section 2 settles notions and notations. In Section 3 the non-Archimedean semimetric is introduced and used to define in a canonical (tree independent) way the reduced game and the Bird core. The relations between stable cost monotonic rules and the Bird core are discussed in Section 4. An axiomatic characterization of the Bird core is given in Section 5. Section 6 concludes.

\section{Preliminaries and notations}

An (undirected) graph is a pair $\langle V, E\rangle$, where $V$ is a set of vertices or nodes and $E$ is a set of edges $e$ of the form $\{i, j\}$ with $i, j \in V, i \neq j$. The complete graph on a set $V$ of vertices is the graph $\left\langle V, E_{V}\right\rangle$, where $E_{V}=\{\{i, j\} \mid i, j \in V$ and $i \neq j\}$. A path between $i$ and $j$ in a graph $<V, E>$ is a sequence of nodes $\left(i_{0}, i_{1}, \ldots, i_{k}\right)$, where $i=i_{0}$ and $j=i_{k}, k \geq 1$, and such that $\left\{i_{s}, i_{s+1}\right\} \in E$ for each $s \in\{0, \ldots, k-1\}$. A cycle in $\langle V, E\rangle$ is a path from $i$ to $i$ for some $i \in V$. A path $\left(i_{0}, i_{1}, \ldots, i_{k}\right)$ is without cycles if there do not exist $a, b \in\{0,1, \ldots, k\}, a \neq b$, such that $i_{a}=i_{b}$.

Two nodes $i, j \in V$ are connected in $\langle V, E\rangle$ if $i=j$ or if there exists a path between $i$ and $j$ in $\langle V, E\rangle$. A connected component of $V$ in $\langle V, E\rangle$ is a maximal subset of $V$ with the property that any two nodes in this subset are connected in $\langle V, E\rangle$. Given a path $P=\left(i_{0}, i_{1}, \ldots, i_{k}\right)$ between $i$ and 
$j$ in a graph $\langle V, E\rangle, k \geq 1$, we say that $v \in V$ is a node in $P$ if $v=i_{m}$ for some $m \in\{0, \ldots, k\}$; we say that an edge $\{r, t\} \in E$ is on the path $P$ or, equivalently, that $i$ is connected to $j$ via the edge $\{r, t\}$ in the path $P$, if there exists $m \in\{0, \ldots, k-1\}$ such that $r=i_{m}$ and $t=i_{m+1}$ or $t=i_{m}$ and $r=i_{m+1}$.

Now, we consider minimum cost spanning tree (mcst) situations. In an mcst situation a set $N=\{1, \ldots, n\}$ of agents is involved willing to be connected as cheap as possible to a source (i.e. a supplier of a service) denoted by 0 . In the sequel we use the notation $N^{\prime}$ for $N \cup\{0\}$. An mcst situation can be represented by a tuple $\left\langle N^{\prime}, E_{N^{\prime}}, w\right\rangle$, where $\left\langle N^{\prime}, E_{N^{\prime}}\right\rangle$ is the complete graph on the set $N^{\prime}$ of nodes or vertices, and $w: E_{N^{\prime}} \rightarrow \mathbb{R}_{+}$is a map which assigns to each edge $e \in E_{N^{\prime}}$ a nonnegative number $w(e)$ representing the weight or cost of edge $e$. We call $w$ a weight function. If $w(e) \in\{0,1\}$ for every $e \in E_{N^{\prime}}$, the weight function $w$ is called a simple weight function, and we refer then to $\left\langle N^{\prime}, E_{N^{\prime}}, w\right\rangle$ as a simple mcst situation. Since in our paper the graph of possible edges is always the complete graph, we simply denote an mest situation with the set of users $N$, source 0 , and weight function $w$ by $\left\langle N^{\prime}, w\right\rangle$. Often we identify an mest situation $\left\langle N^{\prime}, w\right\rangle$ with the corresponding weight function $w$. We denote by $\mathcal{W}^{N^{\prime}}$ the set of all mest situations $\left\langle N^{\prime}, w\right\rangle$ (or $w$ ) with node set $N^{\prime}$. For each $S \subseteq N$ one can consider the mcst subsituation $<S^{\prime}, w_{\mid S^{\prime}}>$, where $S^{\prime}=S \cup\{0\}$ and $w_{\mid S^{\prime}}: E_{S^{\prime}} \rightarrow \mathbb{R}_{+}$is the restriction of the weight function $w$ to $E_{S^{\prime}} \subseteq E_{N^{\prime}}$, i.e. $w_{\mid S^{\prime}}(e)=w(e)$ for each $e \in E_{S^{\prime}}$.

Let $\left\langle N^{\prime}, w\right\rangle$ be an mcst situation. Two nodes $i$ and $j$ are called $\left(w, N^{\prime}\right)$-connected if $i=j$ or if there exists a path $\left(i_{0}, \ldots, i_{k}\right)$ from $i$ to $j$, with $w\left(\left\{i_{s}, i_{s+1}\right\}\right)=0$ for every $s \in\{0, \ldots, k-1\}$. A $\left(w, N^{\prime}\right)$-component of $N^{\prime}$ is a maximal subset of $N^{\prime}$ with the property that any two nodes in this subset are $\left(w, N^{\prime}\right)$-connected. We denote by $C_{i}(w)$ the $\left(w, N^{\prime}\right)$-component to which $i$ belongs and by $\mathcal{C}(w)$ the set of all the $\left(w, N^{\prime}\right)$-components of $N^{\prime}$. Clearly, the collection of $\left(w, N^{\prime}\right)$-components forms a partition of $N^{\prime}$.

We define the set $\Sigma_{E_{N^{\prime}}}$ of linear orders on $E_{N^{\prime}}$ as the set of all bijections $\sigma:\left\{1, \ldots,\left|E_{N^{\prime}}\right|\right\} \rightarrow E_{N^{\prime}}$, where $\left|E_{N^{\prime}}\right|$ is the cardinality of the set $E_{N^{\prime}}$. For each mcst situation $\left\langle N^{\prime}, w>\right.$ there exists at least one linear order $\sigma \in \Sigma_{E_{N^{\prime}}}$ such that $w(\sigma(1)) \leq w(\sigma(2)) \leq \ldots \leq w\left(\sigma\left(\left|E_{N^{\prime}}\right|\right)\right)$. We denote by $w^{\sigma}$ the column vector $\left(w(\sigma(1)), w(\sigma(2)), \ldots, w\left(\sigma\left(\left|E_{N^{\prime}}\right|\right)\right)\right)^{t}$.

For any $\sigma \in \Sigma_{E_{N^{\prime}}}$ we define the set

$$
K^{\sigma}=\left\{w \in \mathbb{R}_{+}^{E_{N^{\prime}}} \mid w(\sigma(1)) \leq w(\sigma(2)) \leq \ldots \leq w\left(\sigma\left(\left|E_{N^{\prime}}\right|\right)\right)\right\},
$$

which we call the Kruskal cone with respect to $\sigma$. One can easily see that $\bigcup_{\sigma \in \Sigma_{E_{N^{\prime}}}} K^{\sigma}=\mathbb{R}_{+}^{E_{N^{\prime}}}$. For each $\sigma \in \Sigma_{E_{N^{\prime}}}$ the cone $K^{\sigma}$ is a simplicial cone 
with generators $e^{\sigma, k} \in K^{\sigma}, k \in\left\{1,2, \ldots,\left|E_{N^{\prime}}\right|\right\}$, where

$$
\begin{gathered}
e^{\sigma, k}(\sigma(1))=e^{\sigma, k}(\sigma(2))=\ldots=e^{\sigma, k}(\sigma(k-1))=0 \\
\text { and } \\
e^{\sigma, k}(\sigma(k))=e^{\sigma, k}(\sigma(k+1))=\ldots=e^{\sigma, k}\left(\sigma\left(\left|E_{N^{\prime}}\right|\right)\right)=1 .
\end{gathered}
$$

[Note that $e^{\sigma, 1}(\sigma(k))=1$ for all $\left.k \in\left\{1,2, \ldots,\left|E_{N^{\prime}}\right|\right\}\right]$. This implies that each $w \in K^{\sigma}$ can be written in a unique way as non-negative linear combination of these generators. To be more concrete, for $w \in K^{\sigma}$ we have

$$
w=w(\sigma(1)) e^{\sigma, 1}+\sum_{k=2}^{\left|E_{N^{\prime}}\right|}(w(\sigma(k))-w(\sigma(k-1))) e^{\sigma, k}
$$

Clearly, we can also write $\mathcal{W}^{N^{\prime}}=\bigcup_{\sigma \in \Sigma_{E_{N^{\prime}}}} K^{\sigma}$, if we identify an mcst situation $<N^{\prime}, w>$ with $w$.

Any mcst situation $w \in \mathcal{W}^{N^{\prime}}$ gives rise to two problems: the construction of a network $\Gamma \subseteq E_{N^{\prime}}$ of minimal cost connecting all users to the source, and a cost sharing problem of distributing this cost in a fair way among users. The cost of a network $\Gamma$ is $w(\Gamma)=\sum_{e \in \Gamma} w(e)$. A network $\Gamma$ is a spanning network on $S^{\prime} \subseteq N^{\prime}$ if for every $e \in \Gamma$ we have $e \in E_{S^{\prime}}$ and for every $i \in S$ there is a path in $\Gamma$ from $i$ to the source. Given a spanning network $\Gamma$ on $N^{\prime}$ we define the set of edges of $\Gamma$ with nodes in $S^{\prime} \subseteq N^{\prime}$ as the set $E_{S^{\prime}}^{\Gamma}=\left\{\{i, j\} \mid\{i, j\} \in \Gamma\right.$ and $\left.i, j \in S^{\prime}\right\}$.

For any mest situation $w \in \mathcal{W}^{N^{\prime}}$ it is possible to determine at least one spanning tree on $N^{\prime}$, i.e. a spanning network without cycles on $N^{\prime}$, of minimum cost; each spanning tree of minimum cost is called an mcst for $N^{\prime}$ in $w$ or, shorter, an mcst for $w$. Two famous algorithms for the determination of minimum cost spanning trees are the algorithm of Prim (Prim (1957)) and the algorithm of Kruskal (Kruskal (1956)). The cost of a minimum cost spanning network $\Gamma$ on $N^{\prime}$ in a simple mest situation $w$ equals $|\mathcal{C}(w)|-1$ (see Lemma 2 in Norde et al. (2004)).

Now, let us introduce some basic game theoretical notations. A cooperative cost game is a pair $(N, c)$ where $N=\{1, \ldots, n\}$ is a finite (player-)set and the characteristic function $c: 2^{N} \rightarrow \mathbb{R}$ assigns to each subset $S \in 2^{N}$, called a coalition, a real number $c(S)$, called the cost of coalition $S$, where $2^{N}$ stands for the power set of the player set $N$, and $c(\emptyset)=0$. The core of a game $(N, c)$ is the set of payoff vectors for which no coalition has an incentive to leave the grand coalition $N$, i.e.

$$
\mathcal{C}(c)=\left\{x \in \mathbb{R}^{N} \mid \sum_{i \in S} x_{i} \leq c(S) \forall S \in 2^{N} \backslash\{\emptyset\} ; \sum_{i \in N} x_{i}=c(N)\right\} .
$$


Note that the core of a game can be empty. A game $(N, c)$ is called a concave game if the marginal contribution of any player to any coalition is more than his marginal contribution to a larger coalition, i.e. if it holds that

$$
c(S \cup\{i\})-c(S) \geq c(T \cup\{i\})-c(T) .
$$

for all $i \in N$ and all $S \subseteq T \subseteq N \backslash\{i\}$.

An order $\tau$ of $N$ is a bijection $\tau:\{1, \ldots,|N|\} \rightarrow N$. This order is denoted by $\tau(1), \ldots, \tau(n)$, where $\tau(i)=j$ means that with respect to $\tau$, player $j$ is in the $i$-th position. We denote by $\Sigma_{N}$ the set of possible orders on the set $N$.

Let $(N, c)$ be a cooperative cost game. For $\tau \in \Sigma_{N}$, the marginal vector $m^{\tau}(c)$ is defined by

$$
m_{i}^{\tau}(c)=c([i, \tau])-c((i, \tau)) \text { for all } i \in N,
$$

where $[i, \tau]=\left\{j \in N: \tau^{-1}(j) \leq \tau^{-1}(i)\right\}$ is the set of predecessors of $i$ with respect to $\tau$ including $i$, and $(i, \tau)=\left\{j \in N: \tau^{-1}(j)<\tau^{-1}(i)\right\}$ is the set of predecessors of $i$ with respect to $\tau$ excluding $i$. In a coherent way with respect to previous notations, we will indicate the set $[i, \tau] \cup\{0\}$ and $(i, \tau) \cup\{0\}$ as $[i, \tau]^{\prime}$ and $(i, \tau)^{\prime}$, respectively. For instance, for each $k \in\{1, \ldots,|N|\}$ and for each $l \in\{2, \ldots,|N|\}$, the set $[\tau(k), \tau]^{\prime}=\{0, \tau(1), \ldots, \tau(k)\}$ and $(\tau(l), \tau)^{\prime}=\{0, \tau(1), \ldots, \tau(l-1)\}$, which will be denoted shorter as $[\tau(k)]^{\prime}$ and $(\tau(l))^{\prime}$, respectively.

Let $\left\langle N^{\prime}, w\right\rangle$ be an mcst situation. The minimum cost spanning tree game $\left(N, c_{w}\right)$ (or simply $c_{w}$ ), corresponding to $\left\langle N^{\prime}, w\right\rangle$, is defined by

$$
c_{w}(S)=\min \left\{w(\Gamma) \mid \Gamma \text { is a spanning network on } S^{\prime}\right\}
$$

for every $S \in 2^{N} \backslash\{\emptyset\}$, with the convention that $c_{w}(\emptyset)=0$.

We denote by $\mathcal{M C S \mathcal { T } ^ { N }}$ the class of all mest games corresponding to mcst situations in $\mathcal{W}^{N^{\prime}}$. For each $\sigma \in \Sigma_{E_{N^{\prime}}}$, we denote by $\mathcal{G}^{\sigma}$ the set $\left\{c_{w} \mid w \in K^{\sigma}\right\}$ which is a cone. We can express $\mathcal{M C S T}^{N}$ as the union of all cones $\mathcal{G}^{\sigma}$, i.e. $\mathcal{M C S} \mathcal{T}^{N}=\bigcup_{\sigma \in \Sigma_{E_{N}}} \mathcal{G}^{\sigma}$, and we would like to point out that $\mathcal{M C S} \mathcal{T}^{N}$ itself is not a cone if $|N| \geq 2$.

The core $\mathcal{C}\left(c_{w}\right)$ of an mcst game $c_{w} \in \mathcal{M C S} \mathcal{T}^{N}$ is nonempty (Granot and Huberman (1981), Bird (1976)) and, given an mest $\Gamma$ (with no cycles) for $N^{\prime}$ in mcst situation $w$, one can easily find an element in the core looking at the Bird allocation in $w$ corresponding to $\Gamma$, i.e. the cost allocation where each player $i \in N$ pays the edge in $\Gamma$ which connects him with his immediate predecessor in $\left\langle N^{\prime}, \Gamma\right\rangle$.

We call a map $F: \mathcal{W}^{N^{\prime}} \rightarrow \mathbb{R}^{N}$ assigning to every mcst situation $w$ a unique cost allocation in $\mathbb{R}^{N}$ a solution. A solution $F$ is efficient if for each 
$w \in \mathcal{W}^{N^{\prime}}$

$$
\sum_{i \in N} F_{i}(w)=w(\Gamma)
$$

where $\Gamma$ is a minimum cost spanning network on $N^{\prime}$ for $w$.

\section{The non-Archimedean semimetric corresponding to an mcst situation}

Let $w \in \mathcal{W}^{N^{\prime}}$. For each path $P=\left(i_{0}, i_{1}, \ldots, i_{k}\right)$ from $i$ to $j$ in the graph $<N^{\prime}, E_{N^{\prime}}>$ we denote the set of its edges by $E(P)$, that is $E(P)=$ $\left\{\left\{i_{0}, i_{1}\right\},\left\{i_{1}, i_{2}\right\}, \ldots,\left\{i_{k-1}, i_{k}\right\}\right\}$. Moreover, we call $\max _{e \in E(P)} w(e)$ the top of the path $P$ and denote it by $t(P)$. We denote by $\mathcal{P}_{i j}^{N^{\prime}}$ the set of all paths without cycles from $i$ to $j$ in the graph $\left\langle N^{\prime}, E_{N^{\prime}}>\right.$.

Now we define the key concept of this section, namely the reduced weight function.

Definition 1 Let $w \in \mathcal{W}^{N^{\prime}}$. The reduced weight function $\bar{w}$ is given by

$$
\bar{w}(i, j)=\min _{P \in \mathcal{P}_{i j}^{N^{\prime}}} \max _{e \in E(P)} w(e)=\min _{P \in \mathcal{P}_{i j}^{N^{\prime}}} t(P)
$$

for each $i, j \in N^{\prime}, i \neq j$.

Now, extending $\bar{w}$ by putting $\bar{w}(i, i)=0$ for each $i \in N^{\prime}$, we obtain a nonnegative function on the set of all pairs of elements in $N^{\prime}$. The obtained reduced weight function $\bar{w}$ is a semimetric on $N^{\prime}$ with the sharp triangle inequality, i.e. a non-Archimedean (NA-) semimetric. In formula, for each $i, j, k \in N^{\prime}$

$$
\begin{aligned}
& \bar{w}(i, j) \geq 0 \text { and } \bar{w}(i, i)=0 \text { (non-negativity); } \\
& \bar{w}(i, j)=\bar{w}(j, i) \text { (symmetry); } \\
& \bar{w}(i, k) \leq \max \{\bar{w}(i, j), \bar{w}(j, k)\} \text { (sharp triangle inequality). }
\end{aligned}
$$

The proof is left to the reader. If $w>0$, then $\bar{w}$ is a non-Archimedean metric on the set $N^{\prime}$.

For the reduced weight function $\bar{w}$ we have a special property related to triangles, as the next lemma shows.

Proposition 1 (The isoscele triangle property) Let $\bar{w}$ be the reduced weight function corresponding to $w \in \mathcal{W}^{N^{\prime}}$ and $i, j, k \in N^{\prime}$ such that $\bar{w}(i, j) \leq$ $\bar{w}(i, k)$ and $\bar{w}(i, j) \leq \bar{w}(k, j)$. Then $\bar{w}(i, k)=\bar{w}(j, k)$. 
Proof By the sharp triangle inequality $\bar{w}(i, k) \leq \max \{\bar{w}(i, j), \bar{w}(j, k)\}=$ $\bar{w}(j, k)$ and $\bar{w}(j, k) \leq \max \{\bar{w}(j, i), \bar{w}(i, k)\}=\bar{w}(i, k)$.

So $\bar{w}(i, k)=\bar{w}(j, k)$.

This property for NA-semimetrics will be useful in proving that there are many minimum cost spanning trees for $\left(N^{\prime}, \bar{w}\right)$, as we see in Theorem 1 .

Unless otherwise clear from the context, in the sequel we simply refer to $\bar{w}$ as the mcst situation which assigns to each edge $\{i, j\} \in E_{N^{\prime}}$ the reduced weight value as defined in equality (??). Further, we will denote by $\overline{\mathcal{W}}^{N^{\prime}} \subset \mathcal{W}^{N^{\prime}}$ the set of all NA-semimetric mest situations which assign to each edge $\{i, j\} \in E_{N^{\prime}}$ the distance $\bar{w}(i, j)$ provided by a NA-semimetric $\bar{w}$ on $N^{\prime}$.

Example 1 Consider the mcst situation $\left\langle N^{\prime}, w>\right.$ with $N^{\prime}=\{0,1,2,3\}$ and $w$ as depicted in Figure 1. Note that $w \in K^{\sigma}$, with $\sigma(1)=\{1,2\}$, $\sigma(2)=\{1,0\}, \sigma(3)=\{1,3\}, \sigma(4)=\{3,0\}, \sigma(5)=\{2,0\}, \sigma(6)=\{2,3\}$. The corresponding mcst situation $\bar{w}$ is depicted in Figure 2.

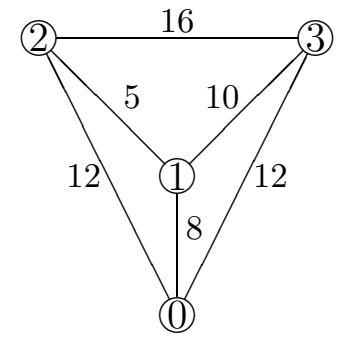

Figure 1: An mcst situation with three agents.

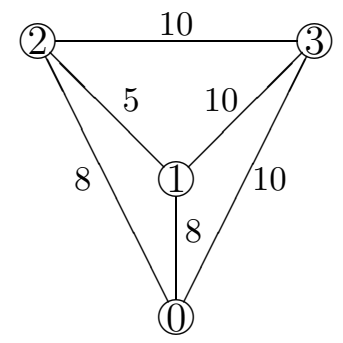

Figure 2: The mest situation $\bar{w}$ corresponding to $w$.

One main result in this section, Proposition 2, concerns an interesting relation which can be established between the mcst situation $\bar{w}$ and a minimal mcst 
situation $w^{\Gamma}$ as defined by Bird (1976), where $\Gamma$ is an mest for $N^{\prime}$ in $w$. Recall that given an mcst situation $w \in \mathcal{W}^{N^{\prime}}$ and an mcst $\Gamma$ for $N^{\prime}$ in $w$, the minimal mest situation $w^{\Gamma}$ is defined (cf. Bird, 1976) by $w^{\Gamma}(\{i, j\})=$ $\max _{e \in P_{i j}^{\Gamma}} w(e)=t\left(P_{i j}^{\Gamma}\right)$, where $P_{i j}^{\Gamma} \in \mathcal{P}_{i j}^{N^{\prime}}$ is the unique path in $\Gamma$ from $i$ to $j$.

Proposition 2 Let $w \in \mathcal{W}^{N^{\prime}}$ and $i, j \in N^{\prime}$. Let $\Gamma$ be an mcst for $N^{\prime}$ in $w$ and $P_{i j}^{\Gamma}$ be the unique path in $\Gamma$ from $i$ to $j$. Then

$$
t\left(P_{i j}^{\Gamma}\right)=\min _{P \in \mathcal{P}_{i j}^{N^{\prime}}} t(P)
$$

Proof Let $P^{*} \in \arg \min _{P \in \mathcal{P}_{i j}^{N^{\prime}}} t(P)$ and let $e^{*}$ be an edge on $P^{*}$ such that $t\left(P^{*}\right)=w\left(e^{*}\right)$. Let $\hat{e}=\{m, n\}$ be an edge on $P_{i j}^{\Gamma}$ with $w(\hat{e})=t\left(P_{i j}^{\Gamma}\right)$.

We have to prove that $w(\hat{e})=w\left(e^{*}\right)$. If so, then it follows immediately that $\min _{P \in \mathcal{P}_{i j}^{N^{\prime}}} t(P)=w\left(e^{*}\right)=w(\hat{e})=t\left(P_{i j}^{\Gamma}\right)$.

If $e^{*}=\hat{e}$, then of course $w\left(e^{*}\right)=w(\hat{e})$.

Otherwise, first note that by definition of $e^{*}$

$$
w(\hat{e}) \geq w\left(e^{*}\right) .
$$

Let $S_{m}$ be the set of all nodes $r \in N^{\prime}$ such that $n$ is not on the path from $r$ to $m$ in $\left\langle N^{\prime}, \Gamma\right\rangle$; let $S_{n}$ be the set of nodes $r \in N^{\prime}$ such that $m$ is not on the path from $r$ to $n$ in $\left\langle N^{\prime}, \Gamma>\right.$, i.e.

$$
S_{m}=\left\{r \in N^{\prime} \mid n \notin P_{m r}^{\Gamma}\right\}
$$

and

$$
S_{n}=\left\{r \in N^{\prime} \mid m \notin P_{n r}^{\Gamma}\right\} .
$$

Note that $\left\{S_{n}, S_{m}\right\}$ is a partition of $N^{\prime}$ and nodes in $S_{n}$ are connected in $<N^{\prime}, \Gamma>$ to nodes in $S_{m}$ via edge $\{m, n\}$. Moreover, by the definition of a path without cycles, $i, j$ must belong to different sets of the partition $\left\{S_{n}, S_{m}\right\}$. So without loss of generality we suppose that $i \in S_{m}$ and $j \in S_{n}$.

Consider the set of edges $E^{+}=\left\{\{t, v\} \mid t \in S_{m}, v \in S_{n}\right\}$. Then,

$$
w(\{m, n\})=w(\hat{e}) \leq w(e), \text { for each } e \in E^{+} .
$$

In order to prove inequality (7), suppose on the contrary that $w(\{m, n\})>$ $w(e)$ for some $e \in E^{+}$. Then the graph $\Gamma^{+}=(\Gamma \backslash\{\hat{e}\}) \cup\{e\}$ would be a spanning network in $N^{\prime}$ cheaper than $\Gamma$, which yields a contradiction.

By the definition of a path, for each $P \in \mathcal{P}_{i j}^{N^{\prime}}$ there exists at least one edge $e \in E^{+}$such that $e$ is on the path $P$. By inequality (7), it follows 
that $t(P) \geq w(e) \geq w(\hat{e})$. This implies that $w\left(e^{*}\right)=\min _{P \in \mathcal{P}_{i j}^{N^{\prime}}} t(P) \geq w(\hat{e})$. Together with inequality (6) we have finally $w\left(e^{*}\right)=w(\hat{e})$.

As a direct consequence of Proposition 2 we have that the mcst situation $\bar{w}$ coincides, for each mest $\Gamma$ for $w$, with the minimal mest situation $w^{\Gamma}$ introduced by Bird (1976). So $w^{\Gamma}=w^{\Gamma^{\prime}}$ for each pair of mest $\Gamma, \Gamma^{\prime}$, a fact which is already known (cf. Aarts (1994), Feltkamp (1995), Feltkamp et al.(1994)), but with a complicated proof.

Let $w \in \mathcal{W}^{N^{\prime}}$ and let $\Gamma$ be an mest for $w$. Let $\tau \in \Sigma_{N}$. We say that $\Gamma$ and $\tau$ fit (or, also, that $\tau$ fits with $\Gamma$ ) if $E_{[\tau(1)]^{\prime}}^{\Gamma}, E_{[\tau(2)]^{\prime}}^{\Gamma}, \ldots, E_{[\tau(|N|)]^{\prime}}^{\Gamma}$ are spanning networks on sets of nodes $[\tau(1)]^{\prime},[\tau(2)]^{\prime}, \ldots,[\tau(|N|)]^{\prime}$, respectively.

Example 2 In Figure 3 is depicted an mcst, denoted by $\Gamma$, for the mcst situation $\bar{w}$ of Figure 2. Consider $\tau_{1}, \tau_{2} \in \Sigma_{N}$ such that $\tau_{1}(1)=1, \tau_{1}(2)=2$,

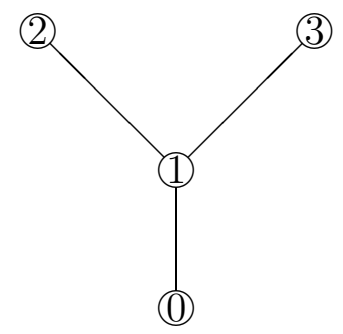

Figure 3: An mest $\Gamma$ for the mest situation $\bar{w}$ of Figure 2.

$\tau_{1}(3)=3$ and $\tau_{2}(1)=1, \tau_{2}(2)=3, \tau_{2}(3)=2$. Note that both $\tau_{1}$ and $\tau_{2}$ fit with $\Gamma$ but none of the other four elements of $\Sigma_{N}$ fit with $\Gamma$.

Remark 1 Let $w \in \mathcal{W}^{N^{\prime}}$, let $\Gamma$ be an mest for $w$ and let $\tau \in \Sigma_{N}$ be an order such that $\Gamma$ and $\tau$ fit. Then,

$$
\sum_{e \in E_{[\tau(r)]^{\top}}^{\Gamma}} w(e)=c_{w}([\tau(r)])
$$

for each $r \in\{1, \ldots,|N|\}$. So $E_{[\tau(r)]^{\prime}}^{\Gamma}$ is an mest for the mest situation $<[\tau(r)]^{\prime}, w_{\mid[\tau(r)]^{\prime}}>$.

Remark 2 Let $w \in \mathcal{W}^{N^{\prime}}$, let $\Gamma$ be an mest for $w$ and let $\tau \in \Sigma_{N}$ be an order such that $\Gamma$ and $\tau$ fit. The marginal vector $m^{\tau}\left(c_{w}\right)$ of the mcst game $c_{w}$ coincides with the Bird allocation in $w$ corresponding to $\Gamma$ and therefore $m^{\tau}\left(c_{w}\right) \in \mathcal{C}\left(c_{w}\right)$, as is proved in Granot and Huberman (1981). 
Remark 3 For each $\sigma \in \Sigma_{E_{N^{\prime}}}$ there exists a tree $\Gamma$ which is an mcst for every $w \in K^{\sigma}$; further, there exists a $\tau \in \Sigma_{N}$ such that $\Gamma$ and $\tau$ fit.

These remarkable considerations together with the next lemma prelude to Theorem 1.

Lemma 1 Let $w \in \overline{\mathcal{W}}^{N^{\prime}}$, let $\Gamma$ be an mcst for $w$ and let $\tau \in \Sigma_{N}$ be such that $\Gamma$ and $\tau$ fit. Let $r \in\{1, \ldots,|N|-1\}$ and let $\tau^{\prime} \in \Sigma_{N}$ be such that $\tau^{\prime}(r)=\tau(r+1), \tau^{\prime}(r+1)=\tau(r)$ and $\tau^{\prime}(i)=\tau(i)$ for each $i \in\{1, \ldots,|N|\} \backslash$ $\{r, r+1\}$ (i.e. $\tau^{\prime}$ is obtained from $\tau$ by a neighbor switch of $\tau(r)$ and $\tau(r+1)$ ). Then there is an mcst $\Gamma^{\prime}$ for $w$ such that $\tau^{\prime}$ and $\Gamma^{\prime}$ fit.

Proof If $\tau(r)$ is not the immediate predecessor of $\tau(r+1)$ in $\Gamma$ then take $\Gamma^{\prime}=\Gamma$ and then $\tau^{\prime}$ and $\Gamma$ fit.

If $\tau(r)$ is the immediate predecessor of $\tau(r+1)$ in $\Gamma$, then let $k \in[\tau(r-1)]^{\prime}$ be the immediate predecessor of $\tau(r)$ in $\Gamma$.

First, note that

$$
w(\{k, \tau(r+1)\}) \geq w(\{k, \tau(r)\})
$$

and

$$
w(\{k, \tau(r+1)\}) \geq w(\{\tau(r), \tau(r+1)\})
$$

because $\Gamma$ is an mest for $w$.

Consider two cases:

c.1) $w(\{k, \tau(r)\}) \leq w(\{\tau(r), \tau(r+1)\})$. Take $\Gamma^{\prime}=(\Gamma \backslash\{\{\tau(r), \tau(r+1)\}\}) \cup$ $\{\{k, \tau(r+1)\}\}$. By inequality (9) and the isoscele triangle property $w(\{k, \tau(r+1)\})=w(\{\tau(r), \tau(r+1)\})$ and then $\Gamma^{\prime}$ is an mcst in $w$ and $\Gamma^{\prime}$ and $\tau^{\prime}$ fit.

c.2) $w(\{\tau(r), \tau(r+1)\})<w(\{k, \tau(r)\})$. Take $\Gamma^{\prime}=(\Gamma \backslash\{\{k, \tau(r)\}\}) \cup$ $\{\{k, \tau(r+1)\}\}$. By inequality (10) and the isoscele triangle property $w(\{k, \tau(r)\})=w(\{k, \tau(r+1)\})$ and then $\Gamma^{\prime}$ is an mcst in $w$ and $\Gamma^{\prime}$ and $\tau^{\prime}$ fit.

Theorem 1 Let $w \in \overline{\mathcal{W}}^{N^{\prime}}$. Then

i) for each $\tau \in \Sigma_{N}$ there exists an mcst $\Gamma$ such that $\Gamma$ and $\tau$ fit. 
ii) Let $c_{w}$ be the mcst game corresponding to $w$. Then $m^{\tau}\left(c_{w}\right) \in \mathcal{C}\left(c_{w}\right)$ for all $\tau \in \Sigma_{N}$ and $c_{w}$ is a concave game.

\section{Proof}

i) Let $\hat{\Gamma}$ be an mest for $w$. Then there is at least one $\hat{\tau} \in \Sigma_{N}$ such that $\hat{\Gamma}$ and $\hat{\tau}$ fit. Further each $\tau$ can be obtained from $\hat{\tau}$ by a suitable sequence of neighbor switches and so, by applying Lemma 1 repeatedly, we obtain the proof.

ii) Let $\Gamma$ be an mest in $N^{\prime}$ for $w$ and let $\tau \in \Sigma_{N}$ such that $\Gamma$ and $\tau$ fit. By Remark 2, it follows that $m^{\tau}\left(c_{w}\right)$ coincides with the Bird allocation corresponding to $\Gamma$. Hence, again by Remark $2, m^{\tau}\left(c_{w}\right) \in \mathcal{C}\left(c_{w}\right)$. Finally, by the Ichiishi theorem (Ichiishi (1981)) telling that a game is concave iff all marginal vectors are in the core of the game, it follows that $c_{w}$ is a concave game.

Let $w \in \mathcal{W}^{N^{\prime}}$. We call the core of the mest game $c_{\bar{w}}$ the Bird core of the mcst game $c_{w}$ and denote it by $\mathcal{B C}(w)$. By Theorem 1 it directly follows that the Bird core $\mathcal{B C}(w)$ of the mest game $c_{w}$ is the convex hull of all the Bird allocations corresponding to the minimum cost spanning trees for $\bar{w}$. Note also that $\mathcal{B C}(w) \subseteq \mathcal{C}\left(c_{w}\right)$, since $c_{\bar{w}}(S) \leq c_{w}(S)$ for each $S \in 2^{N} \backslash\{\emptyset\}$ and $c_{\bar{w}}(N)=c_{w}(N)$ (cf. Feltkamp (1995)).

Example 3 Consider the mcst situation $w$ of Figure 1 and the corresponding reduced mcst situation $\bar{w}$ of Figure 2 . Then

\begin{tabular}{|c|c|c|c|c|c|c|c|}
\hline & $\{1\}$ & $\{2\}$ & $\{3\}$ & $\{1,2\}$ & $\{2,3\}$ & $\{1,3\}$ & $\{1,2,3\}$ \\
\hline \hline$c_{w}$ & 8 & 12 & 12 & 13 & 24 & 18 & 23 \\
\hline$c_{\bar{w}}$ & 8 & 8 & 10 & 13 & 18 & 18 & 23 \\
\hline
\end{tabular}

There are six minimum cost spanning trees for $\bar{w}$. Three of them lead to the Bird allocation $(8,5,10)$ and the other three to the Bird allocation $(5,8,10)$. Further, $m^{\tau}\left(c_{\bar{w}}\right)=(8,5,10)$ for $\tau \in\{(1,2,3),(1,3,2),(3,1,2)\}$ and $m^{\tau}\left(c_{\bar{w}}\right)=$ $(5,8,10)$ for $\tau \in\{(2,1,3),(2,3,1),(3,2,1)\}$. The Bird core $\mathcal{B C}(w)$ is the convex hull of the marginal vectors of the game $c_{\bar{w}}$, that is $\mathcal{B C}(w)=\operatorname{conv}\{(8,5,10)$, $(5,8,10)\} \subset \mathcal{C}\left(c_{w}\right)$.

\section{Monotonicity properties}

In Tijs et al.(2004) a class of solutions for mest situations which are cost monotonic is introduced: the class of obligation rules. Roughly speaking, we 
define a cost monotonic solution for mcst situations as a solution such that, if the costs of some edges increase, then no agent will pay less. More precisely:

Definition 2 A solution $F: \mathcal{W}^{N^{\prime}} \rightarrow \mathbb{R}^{N}$ is a cost monotonic solution if for all mcst situations $w, w^{\prime} \in \mathcal{W}^{N^{\prime}}$ such that $w(e) \leq w^{\prime}(e)$ for each $e \in E_{N^{\prime}}$, it holds that $F(w) \leq F\left(w^{\prime}\right)$.

In this section we introduce a related concept of cost monotonicity for multisolutions on mest situations. We call a correspondence $G: \mathcal{W}^{N^{\prime}} \rightarrow \mathbb{R}^{N}$ assigning to every mcst situation $w$ a set of cost allocations in $\mathbb{R}^{N}$ a multisolution.

Definition 3 A multisolution $M: \mathcal{W}^{N^{\prime}} \rightarrow \mathbb{R}^{N}$ is a cost monotonic multisolution if for all mest situations $w, w^{\prime} \in \mathcal{W}^{N^{\prime}}$ such that $w(e) \leq w^{\prime}(e)$ for each $e \in E_{N^{\prime}}$, it holds that

$$
M(w) \subseteq \operatorname{compr}^{-}\left(M\left(w^{\prime}\right)\right) \quad \text { and } \quad M\left(w^{\prime}\right) \subseteq \operatorname{compr}^{+}(M(w)),
$$

where $\operatorname{compr}^{-}(B)=\left\{x \in \mathbb{R}^{N} \mid \exists b \in B\right.$ s.t. $\left.x_{i} \leq b_{i} \forall i \in N\right\}$ and $\operatorname{compr}^{+}(B)=$ $\left\{x \in \mathbb{R}^{N} \mid \exists b \in B\right.$ s.t. $\left.b_{i} \leq x_{i} \forall i \in N\right\}$, for each $B \subset \mathbb{R}^{N}$.

Before discussing properties of the Bird core as multisolution for mcst situations, we introduce the following propositions dealing with mcst situations originated by NA-semimetrics.

Proposition 3 Let $w \in \overline{\mathcal{W}}^{N^{\prime}}$ and let $\Gamma$ be an mcst for $w$ and $\tau \in \Sigma_{N}$ be such that $\Gamma$ and $\tau$ fit. Then

$$
m_{\tau(j)}^{\tau}\left(c_{w}\right)=\min _{k \in(\tau(j))^{\prime}} w(k, \tau(j)),
$$

for each $j \in\{2, \ldots,|N|\}$.

Proof Let $j \in\{2, \ldots,|N|\}$. Note that by Remark 1

$$
m_{\tau(j)}^{\tau}\left(c_{w}\right)=c_{w}([\tau(j)])-c_{w}((\tau(j)))=\sum_{e \in E_{[\tau(j)]^{\prime}}^{\Gamma}} w(e)-\sum_{e \in E_{(\tau(j))^{\prime}}^{\Gamma}} w(e) .
$$

Since $\Gamma$ and $\tau$ fit, we have $E_{[\tau(j)]^{\prime}}^{\Gamma} \backslash E_{(\tau(j))^{\prime}}^{\Gamma}=\{\{\tau(j), s\}\}$, for some $s \in(\tau(j))^{\prime}$. Because $E_{[\tau(j)]^{\prime}}^{\Gamma}$ is an mcst for $w_{\left[[\tau(j)]^{\prime}\right.}$, we have $s \in \arg \min _{k \in(\tau(j))^{\prime}} w(\{k, \tau(j)\})$. So

$$
\sum_{e \in E_{[\tau(j)]^{\prime}}^{\Gamma}} w(e)-\sum_{e \in E_{(\tau(j))^{\prime}}^{\Gamma}} w(e)=\min _{k \in(\tau(j))^{\prime}} w(k, \tau(j)) .
$$

From (11) and (12) follows the proposition. 
Proposition 4 Let $w, w^{\prime} \in \overline{\mathcal{W}}^{N^{\prime}}$ be NA-semimetric mcst situations such that $w(e) \leq w^{\prime}(e)$ for each $e \in E_{N^{\prime}}$. Then it holds that

$$
m^{\tau}\left(c_{w}\right) \leq m^{\tau}\left(c_{w^{\prime}}\right) \text { for each } \tau \in \Sigma_{N} .
$$

Proof Let $\tau \in \Sigma_{N}$. By Theorem 1 there exist two mcst's $\Gamma$ and $\Gamma^{\prime}$ for $w$ and $w^{\prime}$, respectively, such that they both fit with $\tau$. First note that

$$
m_{\tau(1)}^{\tau}\left(c_{w}\right)=w(0, \tau(1)) \leq w^{\prime}(0, \tau(1))=m_{\tau(1)}^{\tau}\left(c_{w^{\prime}}\right) .
$$

Further

$$
\begin{aligned}
m_{\tau(j)}^{\tau}\left(c_{w}\right) & =\min _{k \in(\tau(j))^{\prime}} w(k, \tau(j)) \\
& \leq \min _{k \in(\tau(j))^{\prime}} w^{\prime}(k, \tau(j)) \\
& =m_{\tau(j)}^{\tau}\left(c_{w^{\prime}}\right)
\end{aligned}
$$

for each $j \in\{2, \ldots,|N|\}$, where the first and the second equality follow by Proposition 3 and the inequality follows from $w(e) \leq w^{\prime}(e)$ for each $e \in E_{N^{\prime}}$.

Theorem 2 The correspondence $\mathcal{B C}$ is a cost monotonic multisolution.

Proof Let $w, w^{\prime} \in \mathcal{W}^{N^{\prime}}$ be such that $w(e) \leq w^{\prime}(e)$ for each $e \in E_{N^{\prime}}$. By Theorem 1 and properties of concave games, $\mathcal{B C}(w)$ is a convex set whose extreme points are the marginal vectors of the game $c_{\bar{w}}$, i.e. each element of $\mathcal{B C}(w)$ is a convex combination of marginal vectors of the game $c_{\bar{w}}$. Let $x \in \mathcal{B C}(w)$. There exist numbers $\alpha^{\tau}, \tau \in \Sigma_{N}$, with $0 \leq \alpha^{\tau} \leq 1$ for each $\tau \in \Sigma_{N}, \sum_{\tau \in \Sigma_{N}} \alpha^{\tau}=1$ and

$$
x=\sum_{\tau \in \Sigma_{N}} \alpha^{\tau} m^{\tau}\left(c_{\bar{w}}\right)
$$

Hence

$$
\begin{aligned}
x & =\sum_{\tau \in \Sigma_{N}} \alpha^{\tau} m^{\tau}\left(c_{\bar{w}}\right) \\
& \leq \sum_{\tau \in \Sigma_{N}} \alpha^{\tau} m^{\tau}\left(c_{\bar{w}^{\prime}}\right) \\
& =x^{\prime} \in \mathcal{B C}\left(w^{\prime}\right),
\end{aligned}
$$

where the inequality follows by Proposition 4 and the fact that $\bar{w}(e) \leq \bar{w}^{\prime}(e)$ for each $e \in E_{N^{\prime}}$ and the second equality by Theorem 1, which proves $\mathcal{B C}(w) \subseteq \operatorname{compr}^{-}\left(\mathcal{B C}\left(w^{\prime}\right)\right)$. Using a similar argument the other way around in relations (14), it follows that $\mathcal{B C}\left(w^{\prime}\right) \subseteq \operatorname{compr}^{+}(\mathcal{B C}(w))$, which concludes the proof.

To connect the cost monotonicity of the Bird core with cost monotonicity of obligation rules, we need Proposition 5. 
Proposition 5 Let $F: \mathcal{W}^{N^{\prime}} \rightarrow \mathbb{R}^{N}$ be a cost monotonic and efficient solution. Then

i) $F(\bar{w})=F(w)$ for every $w \in \mathcal{W}^{N^{\prime}}$;

ii) If $F$ is also stable (i.e. $F\left(w^{\prime}\right) \in \mathcal{C}\left(c_{w^{\prime}}\right)$ for every $\left.w^{\prime} \in \mathcal{W}^{N^{\prime}}\right)$, then $F(w) \in \mathcal{B C}(w)$ for every $w \in \mathcal{W}^{N^{\prime}}$.

Proof Let $w \in \mathcal{W}^{N^{\prime}}$. First note that by Definition 1,

$$
\bar{w}(e) \leq w(e) \text { for each } e \in E_{N^{\prime}} .
$$

Let $\Gamma$ be an mest for $w$.

i) By inequality (15) and cost monotonicity of $F, F(\bar{w}) \leq F(w)$. On the other hand $\Gamma$ is an mest for $\bar{w}$ too and by efficiency of $F$

$$
\sum_{i \in N} F_{i}(\bar{w})=\sum_{i \in N} F_{i}(w)=w(\Gamma)
$$

So, $F(\bar{w})=F(w)$.

ii) By inequality (15),

$$
c_{\bar{w}}(S) \leq c_{w}(S) \text { for all } S \subseteq N,
$$

and by Definition 1

$$
c_{\bar{w}}(N)=c_{w}(N)=w(\Gamma) .
$$

Then by stability of $F, F(\bar{w}) \in \mathcal{C}\left(c_{\bar{w}}\right)=\mathcal{B C}(w) \subseteq \mathcal{C}\left(c_{w}\right)$ and by result (i) $F(w) \in \mathcal{B C}(w)$ too.

Remark 4 Proposition 5 can be extended to multisolutions which are cost monotonic and efficient (Property 1 in next section) multisolutions. From this follows that $\mathcal{B C}$ is the "largest" cost monotonic stable multisolution.

Remark 5 As previously said, in Tijs et al.(2004) we introduced the class of obligation rules and proved that they are both cost monotonic and stable solutions for mcst situations. So, by Proposition 5 it follows that for each $w \in \mathcal{W}^{N^{\prime}}$, the set $\mathcal{F}(w)=\{\phi(w) \mid \phi$ is an obligation rule $\}$ is a subset of the Bird core $\mathcal{B C}(w)$ and $\mathcal{F}(w)=\mathcal{F}(\bar{w})$. 


\section{An axiomatic characterization of the Bird core}

In order to introduce an axiomatic characterization of the Bird core, we need to prove the following fact for NA-semimetric mest situations.

Lemma 2 Let $w, w^{\prime} \in \mathcal{W}^{N^{\prime}}$ and let $\sigma \in \Sigma_{E_{N^{\prime}}}$ be such that $w, w^{\prime} \in K^{\sigma}$. Let $\alpha, \alpha^{\prime} \geq 0$. Then $\alpha \bar{w}, \alpha^{\prime} \bar{w}^{\prime}, \overline{\alpha w+\alpha^{\prime} w^{\prime}} \in K^{\hat{\sigma}}$ for some $\hat{\sigma} \in \Sigma_{E_{N^{\prime}}}$.

Proof By formula (4), for each edge $e \in E_{N^{\prime}}$, there is an edge $\bar{e} \in E_{N^{\prime}}$ such that $\bar{w}(e)=w(\bar{e})$ : given that $e=\{i, j\}, \bar{e}$ is such that $w(\bar{e})=\min _{P \in \mathcal{P}_{i j}^{N^{\prime}}} t(P)$. Note that for each $w_{1}$ in the same cone $K^{\sigma}$ as $w$ we have $\bar{w}_{1}(e)=w(\bar{e})$. This implies that for all pairs of edges $e_{1}, e_{2} \in E_{N^{\prime}}$ :

$$
\bar{w}\left(e_{1}\right) \leq \bar{w}\left(e_{2}\right) \Leftrightarrow w\left(\bar{e}_{1}\right) \leq w\left(\overline{e_{2}}\right) \Leftrightarrow \bar{w}_{1}\left(e_{1}\right) \leq \bar{w}_{1}\left(e_{2}\right) .
$$

So, for each $\bar{\sigma} \in \Sigma_{E_{N^{\prime}}}$ we have:

$$
\bar{w} \in K^{\bar{\sigma}} \Leftrightarrow \bar{w}^{\prime} \in K^{\bar{\sigma}}
$$

Using this fact, respectively, for $\alpha w, \alpha^{\prime} w^{\prime}$ and $\alpha w+\alpha^{\prime} w^{\prime} \in K^{\sigma}$ in the role of $w_{1}$, we obtain:

$$
\bar{w} \in K^{\bar{\sigma}} \Leftrightarrow \alpha \bar{w}, \alpha^{\prime} \bar{w}^{\prime}, \overline{\alpha w+\alpha^{\prime} w^{\prime}} \in K^{\bar{\sigma}},
$$

for each $\bar{\sigma} \in \Sigma_{E_{N^{\prime}}}$.

Proposition 6 Let $w, w^{\prime} \in \mathcal{W}^{N^{\prime}}$ and let $\sigma \in \Sigma_{E_{N^{\prime}}}$ be such that $w, w^{\prime} \in K^{\sigma}$. Let $\alpha, \alpha^{\prime} \geq 0$. Then

i) $\overline{\alpha w+\alpha^{\prime} w^{\prime}}=\alpha \bar{w}+\alpha^{\prime} \bar{w}^{\prime}$;

ii) $c_{\alpha w+\alpha^{\prime} w^{\prime}}=\alpha c_{\bar{w}}+\alpha^{\prime} c_{w^{\prime}}$.

[The NA-semimetric mcst situations $\bar{w}, \overline{w^{\prime}}, \overline{\alpha w+\alpha^{\prime} w^{\prime}}$ are obtained via reduction of the weight functions $w, w^{\prime}, \alpha w+\alpha^{\prime} w^{\prime}$, respectively.]

\section{Proof}

i) Note that

$$
\begin{aligned}
\overline{\alpha w+\alpha^{\prime} w^{\prime}}(\{i, j\}) & =\min _{P \in \mathcal{P}_{i j}^{N^{\prime}}} \max _{e \in E(P)}\left(\alpha w(e)+\alpha^{\prime} w^{\prime}(e)\right) \\
& =\alpha \min _{P \in \mathcal{P}_{i j}^{N^{\prime}}} \max _{e \in E(P)} w(e) \\
& +\alpha^{\prime} \min _{P \in \mathcal{P}_{i j}^{N^{\prime}}} \max _{e \in E(P)} w^{\prime}(e) \\
& =\alpha \bar{w}(\{i, j\})+\alpha^{\prime} \bar{w}^{\prime}(\{i, j\})
\end{aligned}
$$


where the second equality follows from the fact that $w, w^{\prime}$ and $\alpha w+\alpha^{\prime} w^{\prime}$ all belong to $K^{\sigma}$;

ii) Note that, by Lemma 2, $\alpha \bar{w}, \alpha^{\prime} \bar{w}^{\prime}, \overline{\alpha w+\alpha^{\prime} w^{\prime}} \in K^{\bar{\sigma}}$ for some $\bar{\sigma} \in \Sigma_{E_{N^{\prime}}}$. For each $S \in 2^{N} \backslash\{\emptyset\}$, there is, according to Remark 3, a common mcst $\Gamma_{S}$ for $\alpha \bar{w}, \alpha^{\prime} \bar{w}^{\prime}$ and $\overline{\alpha w+\alpha^{\prime} w^{\prime}}$. Hence

$$
\begin{aligned}
\alpha c_{\bar{w}}(S)+\alpha^{\prime} c_{\bar{w}^{\prime}}(S) & =\sum_{e \in \Gamma_{S}} \alpha \bar{w}(e)+\sum_{e \in \Gamma_{S}} \alpha^{\prime} \bar{w}^{\prime}(e) \\
& =\sum_{e \in \Gamma_{S}}\left(\alpha \overline{w^{\prime}}(e)+\alpha^{\prime} \bar{w}(e)\right) \\
& =\sum_{e \in \Gamma_{S}}\left(\overline{\alpha w+\alpha^{\prime} w^{\prime}}(e)\right) \\
& =c_{\overline{\alpha w+\alpha^{\prime} w^{\prime}}}(S)
\end{aligned}
$$

where the third equality follows by $(i)$.

Some interesting properties for multisolutions on mcst situations are the following.

Property 1 The multisolution $G$ is efficient (EFF) if for each $w \in \mathcal{W}^{N^{\prime}}$ and for each $x \in G(w)$

$$
\sum_{i \in N} x_{i}=w(\Gamma)
$$

where $\Gamma$ is a minimum cost spanning network for $w$ on $N^{\prime}$.

Property 2 The multisolution $G$ has the positive (POS) property if for each $w \in \mathcal{W}^{N^{\prime}}$ and for each $x \in G(w)$

$$
x_{i} \geq 0
$$

for each $i \in N$.

Property 3 The multisolution $G$ has the Upper Bounded Contribution (UBC) property if for each $w \in \mathcal{W}^{N^{\prime}}$ and every $\left(w, N^{\prime}\right)$-component $C \neq\{0\}$

$$
\sum_{i \in C \backslash\{0\}} x_{i} \leq \min _{i \in C \backslash\{0\}} w(\{i, 0\})
$$

for each $x \in G(w)$.

Property 4 The multisolution $G$ has the Cone-wise Positive Linearity (CPL) property if for each $\sigma \in \Sigma_{E_{N^{\prime}}}$, for each pair of mcst situations $w, \widehat{w} \in K^{\sigma}$ and for each pair $\alpha, \widehat{\alpha} \geq 0$, we have

$$
G(\alpha w+\widehat{\alpha} \widehat{w})=\alpha G(w)+\widehat{\alpha} G(\widehat{w}) .
$$

[Here we denote by $\alpha G(w)+\widehat{\alpha} G(\widehat{w})$ the set $\{\alpha x+\widehat{\alpha} \widehat{x} \mid x \in G(w), \widehat{x} \in G(\widehat{w}\}$.] 
Proposition 7 The Bird core $\mathcal{B C}$ satisfies the properties EFF, POS, UBC and $C P L$.

Proof Let $w \in \mathcal{W}^{N^{\prime}}$ and let $\sigma \in \Sigma_{E_{N^{\prime}}}$ be such that $w \in K^{\sigma}$. Since $\mathcal{B C}(w)=\mathcal{C}\left(c_{\bar{w}}\right)$, the following considerations hold:

i) For each allocation $x \in \mathcal{B C}(w), \sum_{i \in N} x_{i}=w(\Gamma)$ for some mest $\Gamma$ by the efficiency property of the core of the game $c_{\bar{w}}$. So $\mathcal{B C}$ has the EFF property.

ii) For each allocation $x \in \mathcal{B C}(w), x_{i} \geq 0$ for each $i \in N$ since the Bird core is the convex hull of all Bird allocations in the mest $\bar{w}$, which are vectors in $\mathbb{R}_{+}^{N}$. So $\mathcal{B C}$ has the POS property.

iii) For each $\left(w, N^{\prime}\right)$-component $C \neq\{0\}$ and each $x \in \mathcal{B C}(w)$

$$
\sum_{i \in C \backslash\{0\}} x_{i} \leq c_{\bar{w}}(C \backslash\{0\})=\min _{i \in C \backslash\{0\}} w(\{i, 0\})
$$

by coalitional rationality of the core of the game $c_{\bar{w}}$. So $\mathcal{B C}$ has the UBC property.

iv) Let $\sigma \in \Sigma_{E_{N^{\prime}}}$, let $w, w^{\prime} \in \mathcal{W}^{N^{\prime}}$ be such that $w, w^{\prime} \in K^{\sigma}$ and let $\alpha, \alpha^{\prime} \geq 0$. The core is in fact additive on the class of concave games (see Dragan et al.(1989)). So,

$\mathcal{B C}\left(\alpha w+\alpha^{\prime} w^{\prime}\right)=\mathcal{C}\left(c_{\overline{\alpha w+\alpha^{\prime} w^{\prime}}}\right)=\alpha \mathcal{C}\left(c_{\bar{w}}\right)+\alpha^{\prime} \mathcal{C}\left(c_{\bar{w}^{\prime}}\right)=\alpha \mathcal{B C}(w)+\alpha^{\prime} \mathcal{B C}\left(w^{\prime}\right)$.

Hence $\mathcal{B C}$ has the CPL property.

Inspired by the axiomatic characterization of the $P$-value (Branzei et al.(2004)) we provide the following theorem.

Theorem 3 The Bird core $\mathcal{B C}$ is the largest multisolution which satisfies EFF, POS, UBC and CPL, i.e. for each multisolution $F$ which satisfies $E F F, P O S, U B C$ and $C P L$, we have $F(w) \subseteq \mathcal{B C}(w)$, for each $w \in \mathcal{W}^{N^{\prime}}$.

Proof We already know by Proposition 7 that the Bird core $\mathcal{B C}$ satisfies the four properties EFF, POS, UBC and CPL.

Let $\Psi: \mathcal{W}^{N^{\prime}} \rightarrow \mathbb{R}^{N}$ be a multisolution satisfying EFF, POS, UBC and CPL. Let $w \in \mathcal{W}^{N^{\prime}}$ and $\sigma \in \Sigma_{E_{N^{\prime}}}$ be such that $w \in K^{\sigma}$. We have to prove that 
$\Psi(w) \subseteq \mathcal{B C}(w)$.

First, note that by the CPL property of $\Psi$

$$
\left(w(\sigma(1)) \Psi\left(e^{\sigma, 1}\right)+\sum_{k=2}^{\left|E_{N^{\prime}}\right|}(w(\sigma(k))-w(\sigma(k-1))) \Psi\left(e^{\sigma, k}\right)\right)=\Psi(w) .
$$

Let $x \in \psi(w)$. According to (16) there exists $x^{e^{\sigma, k}} \in \Psi\left(e^{\sigma, k}\right)$ for each $k \in$ $\left\{1, \ldots,\left|E_{N^{\prime}}\right|\right\}$ such that

$$
x=w(\sigma(1)) x^{e^{\sigma, 1}}+\sum_{k=2}^{\left|E_{N^{\prime}}\right|}(w(\sigma(k))-w(\sigma(k-1))) x^{e^{\sigma, k}}
$$

By the UBC property, for each $k \in\left\{1, \ldots,\left|E_{N^{\prime}}\right|\right\}$ and for each $\left(e^{\sigma, k}, N^{\prime}\right)$ component $C \neq\{0\}$ we have

$$
\sum_{i \in C \backslash\{0\}} x_{i}^{e^{\sigma, k}} \leq \min _{i \in C \backslash\{0\}} e^{\sigma, k}(\{i, 0\})= \begin{cases}0 & \text { if } 0 \in C \\ 1 & \text { if } 0 \notin C\end{cases}
$$

implying that

$$
\sum_{i \in N} x_{i}^{e^{\sigma, k}}=\sum_{C \in \mathcal{C}\left(e^{\sigma, k}\right)} \sum_{j \in C \backslash\{0\}} x_{j}^{e^{\sigma, k}} \leq\left|\mathcal{C}\left(e^{\sigma, k}\right)\right|-1=e^{\sigma, k}(\Gamma),
$$

where $\Gamma$ is a minimum spanning network on $N^{\prime}$ for mest situation $e^{\sigma, k}$. By the EFF property, we have $\sum_{i \in N} x_{i}^{e^{\sigma, k}}=e^{\sigma, k}(\Gamma)$, and then inequalities in relation (17) are equalities, that is

$$
\sum_{i \in C \backslash\{0\}} x_{i}^{e^{\sigma, k}}= \begin{cases}0 & \text { if } 0 \in C \\ 1 & \text { if } 0 \notin C .\end{cases}
$$

Now, consider the game $c_{e^{\sigma, k}}$ corresponding to the simple mcst situation $\overline{e^{\sigma, k}}$. Note that for each $S \in 2^{N} \backslash\{\emptyset\}$,

$$
c_{e^{\sigma, k}}(S)=\mid\left\{C: C \text { is a }\left(e^{\sigma, k}, N^{\prime}\right)-\text { component, } C \cap S \neq \emptyset, 0 \notin C\right\} \mid,
$$

which is the number of $\left(e^{\sigma, k}, N^{\prime}\right)$-components not connected to 0 in $e^{\sigma, k}$ with at least one node in the player set $S$. 
By (18) and the POS property, it follows that $\sum_{i \in S} x_{i}^{e^{\sigma, k}} \leq c_{e^{\sigma, k}}(S)$ and together with the EFF property we have $x^{e^{\sigma, k}} \in \mathcal{C}\left(c_{e^{\sigma, k}}\right)=\mathcal{B C}\left(e^{\sigma, k}\right)$. Moreover, from Proposition 6 it follows

$$
x=\left(w(\sigma(1)) x^{e^{\sigma, 1}}+\sum_{k=2}^{\left|E_{N^{\prime}}\right|}(w(\sigma(k))-w(\sigma(k-1))) x^{e^{\sigma, k}}\right) \in \mathcal{C}\left(c_{\bar{w}}\right)=\mathcal{B C}(w) .
$$

Keeping into account relation $(16)$, we have $\Psi(w) \subseteq \mathcal{B C}(w)$.

\section{Final remarks}

This paper deals mainly with the monotonicity and additivity properties of the Bird core. The attention to monotonicity properties of solutions for cost and reward sharing situations is growing in the literature.

In Sprumont (1990) attention is paid to population monotonic allocations schemes (pmas), in Branzei et al.(2001) and Voorneveld et al.(2002) to bimonotonic allocation schemes (bi-mas) and in Branzei et al.(2002) to type monotonic allocation schemes. For mcst-situations, the existence of population monotonic allocation schemes was established in Norde et al.(2004). For special directed mcst-situations also pmas-es exists as is shown in Moretti et al.(2002).

In Tijs et al.(2004) so called obligation rules for mcst-situations turn out to be cost monotonic and induce also a pmas. A special obligation rule is the $P$-value discussed in Branzei et al.(2004) (see also Feltkamp et al.(1994), Feltkamp (1995)). The $P$-value can be seen as a special selection of the Bird core: it corresponds to the barycenter of the Bird core (cf. Moretti et al.(2004), Bergañtinos and Vidal-Puga (2004)).

For additivity properties of solutions we refer to Branzei and Tijs (2001), Tijs and Branzei (2002).

\section{References}

Aarts, H., 1994. Minimum cost spanning tree games and set games, $\mathrm{PhD}$ Dissertation, University of Twente, The Netherlands.

Bird, C.G., 1976. On cost allocation for a spanning tree: a game theoretic approach, Networks 6, 335-350. 
Bergañtinos, G., Vidal-Puga, J.J., 2004. Defining rules in cost spanning tree problems through the canonical form, EconPapers, RePEc:wpa:wuwpga: 0402004 .

Branzei, R., Moretti, S., Norde, H., Tijs, S., 2004. The P-value for cost sharing in minimum cost spanning tree situations, Theory and Decision 56, 47-61.

Branzei R., Solymosi T., Tijs, S., 2002. Type monotonic allocation schemes for multi-glove games, CentER DP 2002-117, Tilburg University, The Netherlands.

Branzei R., Tijs, S., 2001. Additivity regions for solutions in cooperative game theory, Libertas Mathematica 21, 155-167.

Branzei, R., Tijs, S., Timmer, J., 2001. Information collecting situations and bi-monotonic allocation schemes, Mathematical Methods of Operations Research 54, 303-313.

Claus, A., Kleitman, D.J., 1973. Cost allocation for a spanning tree, Networks 3, 289-304.

Dragan, I., Potters, J., Tijs, S., 1989. Superadditivity for solutions of coalitional games, Libertas Mathematica 9, 101-110.

Feltkamp, V., 1995. Cooperation in controlled network structures, PhD Dissertation, Tilburg University, The Netherlands.

Feltkamp, V., Tijs, S., Muto, S., 1994. On the irreducible core and the equal remaining obligations rule of minimum cost spanning extension problems, CentER DP 106, Tilburg University, The Netherlands.

Granot, D., Claus, A., 1976. Game theory application to cost allocation for a spanning tree. Working Paper 402, Faculty of Commerce and Business Administration, University of British Columbia.

Granot, D., Huberman, G., 1981. On minimum cost spanning tree games, Mathematical Programming 21, 1-18.

Ichiishi, T., 1981. Super-modularity: applications to convex games and the 
greedy algorithm for LP, Journal of Economic Theory 25, 283-286.

Kruskal, J.B., 1956. On the shortest spanning subtree of a graph and the traveling salesman problem, Proceedings of the American Mathematical Society $7,48-50$.

Moretti, S., Norde, H., Pham Do, K.H., Tijs, S., 2002. Connection problems in mountains and monotonic allocation schemes, Top 10, 83-99.

Moretti, S., Tijs, S., Branzei, R., Norde, H., 2004. Conservative construct and charge rules for minimum cost spanning tree situations, Working Paper.

Norde, H., Moretti, S., Tijs, S., 2004. Minimum cost spanning tree games and population monotonic allocation schemes, European Journal of Operational Research 154, 84-97.

Prim, R.C., 1957. Shortest connection networks and some generalizations, Bell Systems Technical Journal 36, 1389-1401.

Sprumont, Y., 1990. Population monotonic allocation schemes for cooperative games with transferable utility, Games and Economic Behavior 2, 378-394.

Tijs, S., Branzei, R., 2002. Additive stable solutions on perfect cones of cooperative games, International Journal of Game Theory 31, 469-474.

Tijs, S., Branzei, R., Moretti, S., Norde, H., 2004. Obligation rules for minimum cost spanning tree situations and their monotonicity properties, CentER DP 2004-53, Tilburg University, The Netherlands.

Voorneveld, M., Tijs, S. and Grahn, S., 2002. Monotonic allocation schemes in clan games, Mathematical Methods of Operations Research 56, 439-449. 